\title{
Novel LC Method Development and Validation for Simultaneous Determination of Montelukast and Doxofylline in Bulk and Pharmaceutical Dosage Forms
}

\author{
Gadapa Nirupa, ${ }^{1}$ A. Siva Kumar, ${ }^{2}$ and Upendra M. Tripathi ${ }^{2}$ \\ ${ }^{1}$ Department of Chemistry, Jawaharlal Nehru Technological University, Kukatpally, Hyderabad 500 085, India \\ ${ }^{2}$ Analytical R\&DD Department, Startech Labs Pvt Ltd, 2nd Floor, SMR Chambers, H. No. 1-58/7 Madinaguda, \\ Hyderabad 500 050, India
}

Correspondence should be addressed to Upendra M. Tripathi; drtripathi@startechlabs.com

Received 6 June 2012; Accepted 10 July 2012

Academic Editor: Irene Panderi

Copyright (C) 2013 Gadapa Nirupa et al. This is an open access article distributed under the Creative Commons Attribution License, which permits unrestricted use, distribution, and reproduction in any medium, provided the original work is properly cited.

\begin{abstract}
A novel rapid HPLC method was developed for simultaneous determination of montelukast and doxofylline in bulk and pharmaceutical dosage forms. Development of an analytical method for simultaneous estimation of drugs requires a lot of efforts and of course it is a challenging task. The method was developed by using $\mathrm{C} 18(150 \mathrm{~mm} \times 4.6 \mathrm{~mm}, 5 \mu \mathrm{m})$ column; mobile phase consisting of methanol and phosphate buffer at $\mathrm{pH} 4.5$; the flow rate of $1.0 \mathrm{~mL} / \mathrm{min}$ and ultraviolet detection at $280 \mathrm{~nm}$. Both drugs were sufficiently resolved having retention time of $4.7 \mathrm{~min}$ and $1.9 \mathrm{~min}$ for montelukast and doxofylline, respectively. The method was validated as per ICH Guidelines for various parameters like precision, linearity, accuracy, ruggedness, and robustness. The validated method was applied to the commercially available pharmaceutical dosage form and obtained the desired result.
\end{abstract}

\section{Introduction}

In continuation to our earlier efforts $[1,2]$ to support our pharmaceutical fraternity, in this paper also we are trying to contribute with the analytical method of very important drugs required to cure most common disease, asthma.

Asthma is a chronic inflammatory disease that affects children and adults with symptoms such as a runny nose, nasal congestion, and sneezing. It causes inflammation of the airways in which the smaller passages of the lungs narrow. This causes wheezing, shortness of breath, mucus production, and coughing. With the present day's life style and due to environmental pollution, the population of asthma-infected people is drastically increasing. Hence, it occupies great medical importance. This can be controlled using anti-inflammatory drugs, bronchodilators, or leukotriene modifiers. Due to this reason, we have selected two important drugs, namely, montelukast and doxofylline for the study. Earlier, one method development for single drug, montelukast, using HPLC has been executed and submitted as a part of the author's dissertation work [3]. It has encouraged the authors to extend this study with multidrug matrix. In the present study, two drugs montelukast and doxofylline combination has been selected, which are used in the medication and maintenance of asthma.

Montelukast is a leukotriene receptor antagonist (LTRA) used for the maintenance treatment of asthma and to relieve symptoms of seasonal allergies. It is usually administered orally. Montelukast is a CysLT ${ }_{1}$ antagonist and it blocks the action of leukotriene D4 (and secondary ligands LTC4 and LTE4) on the cysteinyl leukotriene receptor CysLT in the lungs and bronchial tubes by binding to it. This reduces the bronchoconstriction otherwise caused by the leukotriene and results in less inflammation. Decreasing inflammation reduces swelling that narrows airways. Montelukast also relaxes bronchial tube walls. Apart from being used to treat asthma, it is also used to treat symptoms of hay fever and allergic rhinitis. Montelukast sodium is described chemically as [R-(E)]-1-[[[1-[3-[2-(7-chloro2 quinolinyl) ethenyl] phenyl]-3-[2-(1-hydroxy-1-methyl ethyl)phenyl]propyl] thio]methyl]cyclopropane acetic acid, monosodium salt. 


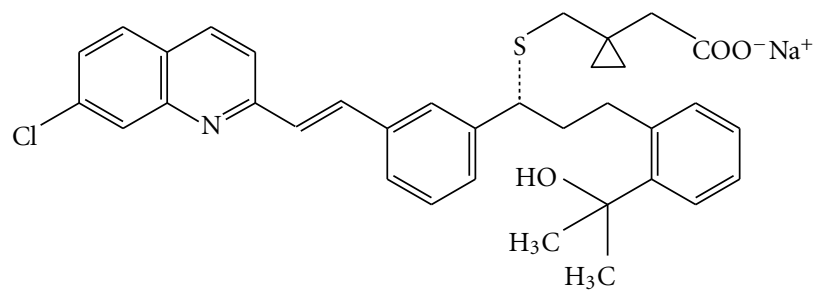

(a) Montelukast

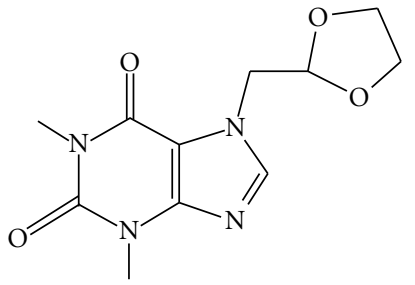

(b) Doxofylline

FIgURE 1: Chemical structure of the drugs.

Doxofylline (also known as doxophylline) is a xanthine derivative drug used in the treatment of asthma. It has antitussive and bronchodilator effects and acts as a phosphodiesterase inhibitor. Bronchodilators are drugs used to relieve the symptoms of an asthma attack by clearing mucus from the lungs and reducing swollen airways. Doxofylline is used to treat the symptoms of asthma as well as certain other lung diseases and conditions. It is proven to alleviate symptoms by reducing the urge to cough and helping to increase the airflow to the lungs. Doxofylline is chemically described as 7-(1,3-dioxolan-2-ylmethyl)-1,3dimethylpurine-2,6-dione. The chemical structures of montelukast and doxofylline are shown in the Figure 1.

For better maintenance therapy in patients suffering from asthma and chronic obstructive pulmonary disease (COPD), Montelukast and Doxofylline are used in combination. This drug combination is also available in the market. Hence, these drugs are selected for the analytical studies.

For individual estimation of these drugs, several methods are available in the literature [4-15]. Some methods which are available in literature are for the simultaneous estimation of montelukast and levocetirizine [16-19]. Some other methods available in the literature are for the simultaneous estimation of montelukast and bambuterol [20-22]. There is only one method [23] available in the literature for the simultaneous estimation of montelukast and doxofylline and that too has certain limitations. The present method of this paper is faster and user friendly than the method available in the literature for the simultaneous estimation of Montelukast and Doxofylline.

\section{Experimental Section}

2.1. Method Development. Selection of UV Maxima. A dilute solution of Montelukast showed the UV maxima at $286 \mathrm{~nm}$ and the absorbance has been found to be very poor due to less chromophoric nature of the compound. On the other hand, Doxofylline has exhibited the UV maxima at $273 \mathrm{~nm}$, and $205 \mathrm{~nm}$ and the response was very good. To undertake the combined estimation of both drugs, it has been decided to select the UV maxima of $280 \mathrm{~nm}$, which falls between the maxima of both the drugs and seems to be suitable.

Selection of Column. As C18 is the most widely used column, therefore, we decided to develop a method on a common column so that anybody can utilize this method for their application.

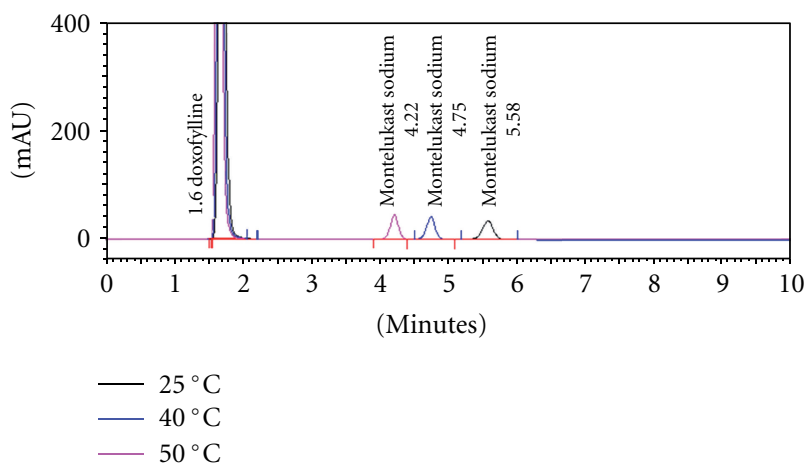

FIGURE 2: Temperature variation chromatograms.

Selection of the Mobile Phase Composition. Various mobile phase compositions have been tried to establish the simultaneous estimation of both the drugs. We tried with the simple mixture of methanol and water, which was followed up with the combinations of various Buffers but out of these the combination of methanol and phosphate buffer has been found to be suitable. Buffer $\mathrm{pH}$ has also been optimized by performing the estimation at various $\mathrm{pH}$, namely, 3.0, 4.5, and 7.5 and after evaluation of the peak shape and retention time, it has been concluded to adopt the buffer $\mathrm{pH}$ of 4.5 .

Selection of Column Temperature. This study was quite interesting, and the determination of the combined drugs has been performed at three different temperatures, namely, $25^{\circ} \mathrm{C}, 40^{\circ} \mathrm{C}$ and $50^{\circ} \mathrm{C}$. It has been found that the retention time of Doxofylline was unchanged $(1.6 \mathrm{~min})$ at all temperatures, whereas, the retention time of Montelukast has been found to be variable at different temperatures as $5.58 \mathrm{~min}$ at $25^{\circ} \mathrm{C}, 4.75$ $\min$ at $40^{\circ} \mathrm{C}$, and $4.22 \mathrm{~min}$ at $50^{\circ} \mathrm{C}$. The representative graph is shown in Figure 2. Actually, $50^{\circ} \mathrm{C}$ is not a very suitable temperature for a routine analysis. So it was not selected for carrying out the analysis. We observed the difference of retention time of Montelukast between analysis at $40^{\circ} \mathrm{C}$ and $25^{\circ} \mathrm{C}$ to be almost $1 \mathrm{~min}$. This was quite encouraging. Therefore, we decided to adopt $40^{\circ} \mathrm{C}$ of analysis temperature to speed up the analysis and elute both the peaks below $5 \mathrm{~min}$, which is really demonstrating for the rapid estimation of this combination drug.

Optimised Method of Analysis and Other Chromatographic Conditions Are Below. The Chromatographic column, C18 $(150 \mathrm{~mm} \times 4.6 \mathrm{~mm}, 5 \mu \mathrm{m})$ was used as a stationary phase. 

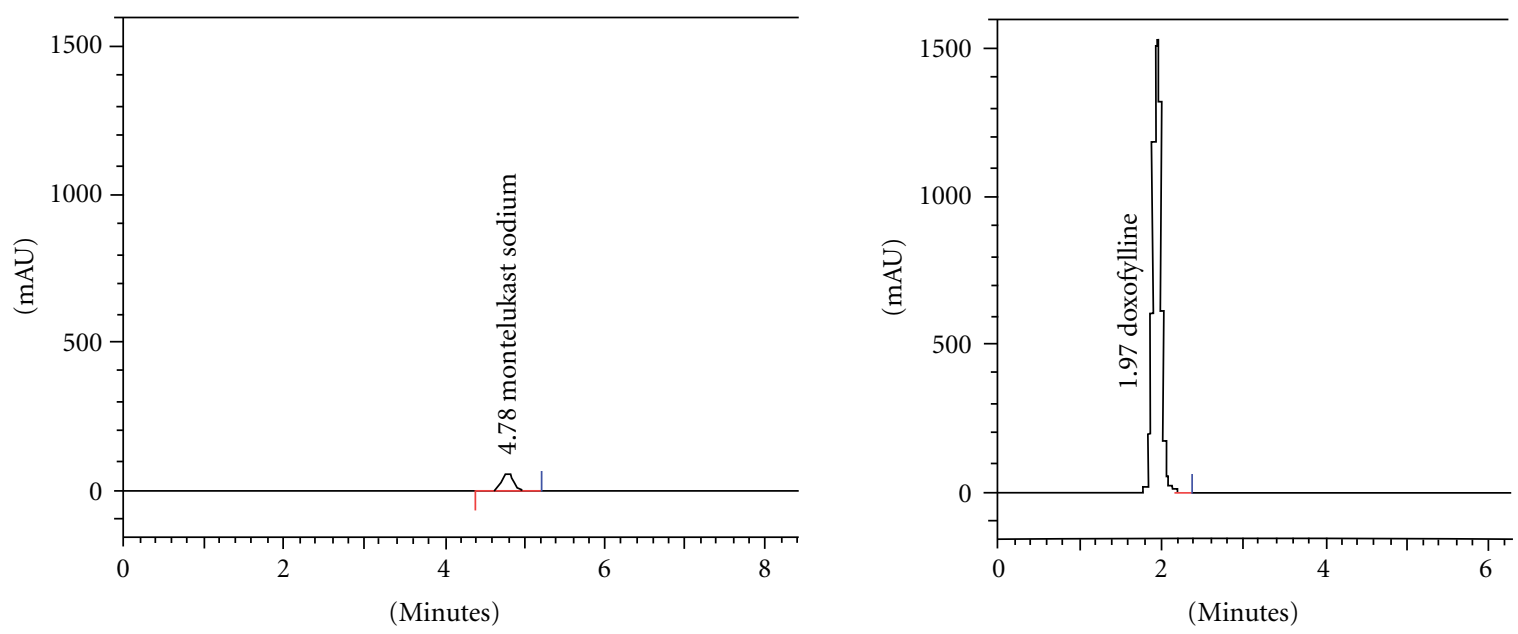

FIgURE 3: Individual drugs.

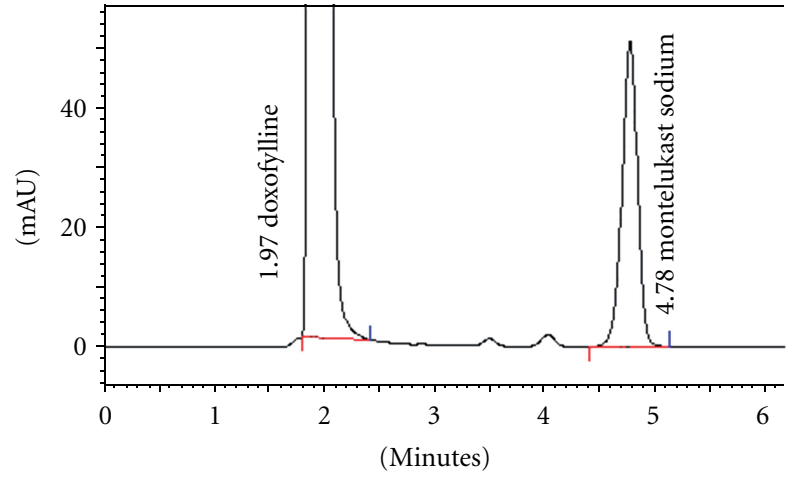

FIGURE 4: Drug mixture.

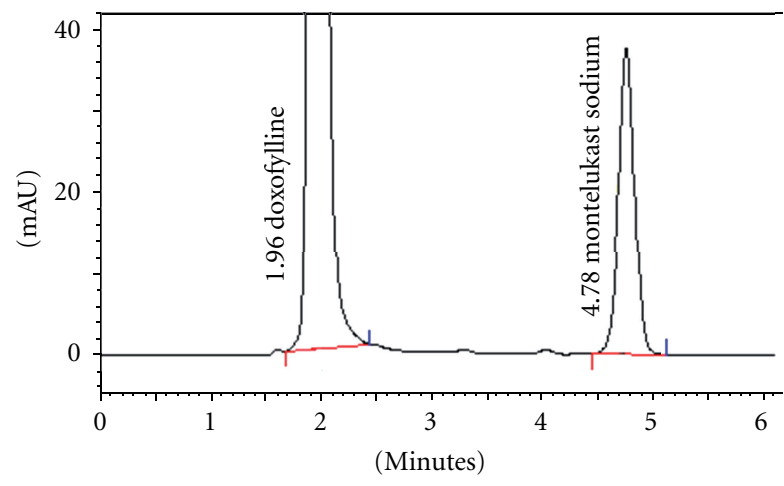

FIGURE 5: Dosage form.

Mobile phase was prepared with Buffer and Methanol (10: 90). Buffer was prepared by dissolving $0.01 \mathrm{M}$ Sodium Hydrogen orthophosphate in $1000 \mathrm{ML}$ of water. The $\mathrm{pH}$ were adjusted to 4.5 with dilute OrthoPhosphoric acid. The diluents used for the analysis was prepared with Methanol and Water $(80: 20)$. Injection volume was $20 \mu \mathrm{L}$. The pump flow rate was $1.0 \mathrm{~mL} / \mathrm{min}$. The column temperature was maintained at $40^{\circ} \mathrm{C}$. The eluent was detected at $280 \mathrm{~nm}$. The run time was $20 \mathrm{~min}$.

2.2. Materials, Reagents, and Chemicals. Montelukast and Doxofylline Standards were obtained from Startech Labs. Combination drug tablets used for the experiment, TelekastD, was manufactured by Intas Pharmaceuticals. HPLC grade Methanol, Sodium Phosphate, and Orthophosphoric acid were obtained from Merck, Darmstadt, Germany.

2.3. Equipments. UV Visible spectrophotometer used was Shimadzu, model UV-2450. The HPLC instrument used was Schimadzu make, model-LC-2010 CHT. Class VP Software was used for data acquisition.

2.4. Preparation of Standard Solution. Mixed standard solution of $0.01 \mathrm{mg} / \mathrm{mL}$ of Montelukast and $0.4 \mathrm{mg} / \mathrm{mL}$ of Doxofylline (treat this as $100 \%$ for various experimental purposes) was prepared by taking $25 \mathrm{mg}$ of Montelukast and dissolving in $25 \mathrm{~mL}$ diluents. $1 \mathrm{~mL}$ of this solution was mixed with $40 \mathrm{mg}$ of Doxofylline in $100 \mathrm{~mL}$ volumetric flask diluted upto the mark with the diluent.

2.5. Preparation of Linearity Solutions. For Linearity $150 \%$, $125 \%, 100 \%, 75 \%$, and $50 \%$ solutions were prepared.150\% solution was prepared by dissolving $25 \mathrm{~mL}$ of Montelukast in $25 \mathrm{~mL}$ of diluent. $1.5 \mathrm{~mL}$ of this solution was added to $60 \mathrm{mg}$ of Doxofylline and dissolved in $100 \mathrm{~mL}$ diluent. $20.83 \mathrm{~mL}$ of $150 \%$ solution was dissolved in $25 \mathrm{~mL}$ diluent for preparing $125 \%$ solution. $16.66 \mathrm{~mL}$ of $150 \%$ solution was dissolved in $25 \mathrm{~mL}$ diluent for preparing 100\% solution. $12.5 \mathrm{~mL}$ of $150 \%$ solution was dissolved in $25 \mathrm{~mL}$ diluent for preparing $75 \%$ solution. $8.33 \mathrm{~mL}$ of $150 \%$ solution was dissolved in $25 \mathrm{~mL}$ diluent for preparing $50 \%$ solution.

2.6. Sample Preparation for Accuracy. Three different solutions were prepared for performing the accuracy studies. The first solution was prepared by dissolving $25 \mathrm{mg}$ of Montelukast in $25 \mathrm{~mL}$ diluent. $1 \mathrm{~mL}$ of this solution was added to 
TABLE 1: System suitability results.

\begin{tabular}{lcccc}
\hline Standard & & Average & SD & \%RSD \\
\hline & Retention time & 4.79 & 0.002449 & 0.05 \\
Montelukast & Area & 507292.8 & 4646.642788 & 0.91 \\
& Resolution & 12.414 & 0.074699398 & 88.82016032 \\
& Theoretical plates & 5660.126 & 0.017888544 & 1.56 \\
\hline \multirow{3}{*}{ Doxofylline } & Asymmetry & 0.948 & 0.000837 & 0.04 \\
& Retention time & 1.97 & 11488.81385 & 0.099 \\
& Area & 11536096.2 & 0 & 0.75 \\
& Resolution & 0 & 11.65102442 & 0.004472136 \\
\hline
\end{tabular}

TABLE 2: Linearity results.

\begin{tabular}{lcc}
\hline & Linearity range & Correlation coefficient \\
\hline Montelukast & $0.005-0.015 \mathrm{mg} / \mathrm{mL}$ & 0.9941 \\
Doxofylline & $0.2-0.6 \mathrm{mg} / \mathrm{mL}$ & 0.9935 \\
\hline
\end{tabular}

$40 \mathrm{mg}$ of Doxofylline in $100 \mathrm{~mL}$ volumetric flask and made up the solution with $150 \%$ linearity solution. The second solution was prepared by dissolving $25 \mathrm{mg}$ of Montelukast in $25 \mathrm{ml}$ diluent. $1 \mathrm{~mL}$ of this solution was added to $40 \mathrm{mg}$ of Doxofylline in $100 \mathrm{~mL}$ volumetric flask and make up the solution with $100 \%$ linearity solution. The third solution was prepared by dissolving $25 \mathrm{mg}$ of Montelukast in $25 \mathrm{ml}$ diluent. $1 \mathrm{~mL}$ of this solution was added to $40 \mathrm{mg}$ of Doxofylline in $100 \mathrm{~mL}$ volumetric flask and make up the solution with $50 \%$ linearity solution.

2.7. Preparation of Sample Solution for Batch Analysis. The method was applied to the analysis of commercial sample, Telekast-D. The average weight of ten tablets was calculated and found to be $644.93 \mathrm{mg}$. The tablet was crushed to a homogeneous mixture and $59.43 \mathrm{mg}$ of the tablet was dissolved in $100 \mathrm{~mL}$ of the mobile phase. The solution was sonicated for 5 minutes followed by cyclomixing for 5 minutes to extract the drug in solution. The resulting solution was filtered by using Millipore syringe filter $(0.42 \mu)$. The resulting clear solution was injected in HPLC in duplicate as per the developed method.

\subsection{Analytical Method Validation}

2.8.1. Specificity of the Method. Specificity is the ability of the method to measure the analyte response in the presence of its potential impurities. This parameter was performed to know the Retention Time of each drug in a mixture and in the sample to understand if any chromatographic interference between the analytes or drug-excipient interference is present.

2.8.2. System Suitability. System suitability test is used to verify if the resolution and reproducibility of the chromatographic systems are adequate for the analysis to be done. The tests are based on the fact that the equipment, electronics, samples to be analyzed constitute an integral system that can be evaluated as such. The limits for system suitability were set for theoretical plates, resolution, and asymmetry.

2.8.3. Linearity. Concentrations of the standard mixture, $50 \%, 75 \%, 100 \%, 125 \%$, and $150 \%$ were prepared and injected, and chromatogram was recorded. A graph was plotted for the concentration of the corresponding drug versus area. The Correlation coefficient ( $r$ ) for each drug was calculated.

2.8.4. Accuracy. To determine the accuracy in sample preparation, method of standard additions was made for measuring the recovery of the drugs. To the known standard solution concentrations of the drug (50\%, 100\%, and 150\%) were added. Three different solutions were prepared as mentioned in Section 2.6. The accuracy was expressed as the percentage of the analyte recovery.

2.8.5. Robustness. To verify the robustness of the method, slight changes to the chromatographic conditions were made to check for any changes in the chromatogram. The analysis was done under variable flow rates, wave lengths, $\mathrm{pH}$, and temperatures. The flow rate as per the developed method was $1 \mathrm{~mL} / \mathrm{min}$. This has been purposely changed to $0.8 \mathrm{~mL} / \mathrm{min}$ and $1.2 \mathrm{~mL} / \mathrm{min}$, and the chromatogram was obtained. The wave length as per the developed method was $280 \mathrm{~nm}$. This has been purposely changed to $278 \mathrm{~nm}$ and $282 \mathrm{~nm}$. The $\mathrm{pH}$ in the developed method was 4.5 . This was changed to 4.3 and 4.7 , and the chromatogram was obtained. The column temperature in the developed method was $40^{\circ} \mathrm{C}$. This was changed to $39.5^{\circ} \mathrm{C}$ and $40.5^{\circ} \mathrm{C}$, and the chromatogram was obtained.

2.8.6. Ruggedness. To verify the ruggedness of the method, the analysis was done on different days and different chemists to check for any changes in the chromatogram. The percentage RSD for the retention time and area was calculated.

2.8.7. Performance of the Method on Commercial Samples. The method is said to be effective if it can be applied for the analysis of commercial tablets. For this purpose, performance 
TABLE 3: Results for the accuracy of the method.

\begin{tabular}{|c|c|c|c|c|c|c|}
\hline & & Initial conc area & Sol 1 area & $50 \%$ area & Sol $1-50 \%$ area & \%Recovery \\
\hline \multirow{3}{*}{ Sol 1} & Montelukast & 494506 & 785653 & 295518 & 291177 & 98.53 \\
\hline & Doxofylline & 10344817 & 16526064 & 6302308 & 6181247 & 99.56 \\
\hline & & Initial conc area & Sol 2 area & $100 \%$ area & Sol $2-75 \%$ area & \%Recovery \\
\hline \multirow{3}{*}{ Sol 2} & Montelukast & 494506 & 988418 & 494506 & 493912 & 99.88 \\
\hline & Doxofylline & 10344817 & 20787092 & 10344817 & 10442275 & 100.94 \\
\hline & & Initial conc area & Sol 3 area & $150 \%$ area & Sol 3-100\% area & \%Recovery \\
\hline \multirow{2}{*}{ Sol 3} & Montelukast & 494506 & 1201875 & 712264 & 707369 & 99.31 \\
\hline & Doxofylline & 10344817 & 25046850 & 14537835 & 14702033 & 101.13 \\
\hline
\end{tabular}

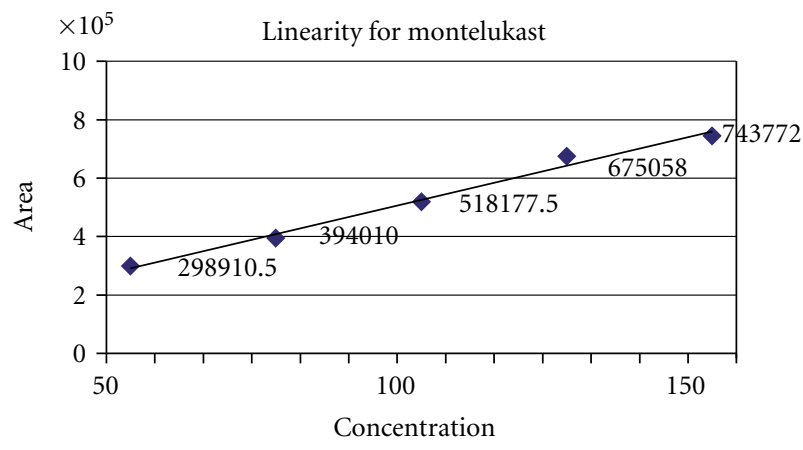

(a)

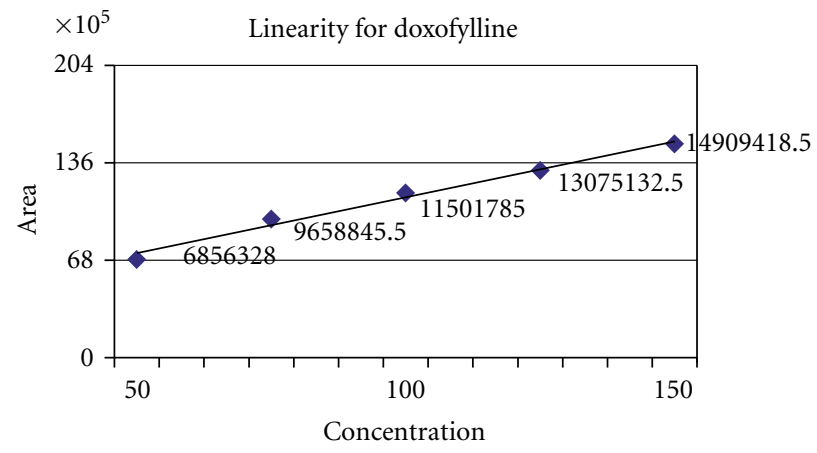

(b)

FIGURE 6: Graphs showing linearity of the drugs.

test of the method has been conducted on the market sample Telekast-D, Batch no. 06TTD040 manufactured by Intas Pharmaceuticals.

\section{Results and Discussion}

The present work involves simultaneous estimation of Montelukast and Doxofylline using reverse phase HPLC. The current trend followed by the industries is developing a methodology which can save sophisticated instruments and chemist's valuable time by which the product analysis can be done very fast, thereby saving the time. This is the reason why people are more attracted towards UFLC (Ultra-Fast Liquid Chromatography) $[24,25]$ methods, though most of the pharmacopeia still have the HPLC methods only.

Keeping all these points in mind, the current method has been developed and it is very fast and encouraging. The developed method was validated with a holistic approach according to ICH guidelines and details of findings are expressed in what follows.

3.1. Specificity of the Method. The Retention times of the standard drugs individually were measured and it was found to be $4.78 \mathrm{~min}$ and $1.97 \mathrm{~min}$ for Montelukast and Doxofylline, respectively. The drugs were mixed and injected for taking the chromatogram. Both drugs were resolved sufficiently in the mixture. Retention time of both drugs in standard mix was found to be $4.78 \mathrm{~min}$ and $1.97 \mathrm{~min}$ for Montelukast and Doxofylline, respectively. This indicates that there is no chromatographic interference between the analytes. The pharmaceutical dosage form (tablet) was then injected, and the chromatogram was obtained. The retention time of the drugs in the dosage form was found to be $4.78 \mathrm{~min}$ and $1.96 \mathrm{~min}$ for Montelukast and Doxofylline, respectively. There is no specific change in retention time of both drugs, which indicates that there is no drug-excipient interference. Respective HPLC chromatograms are represented in Figures 3, 4 and 5.

The resolution between Montelukast and Doxofylline was 12.36 which indicates a very good separation. The asymmetry factor for Montelukast and Doxofylline was 0.95 and 0.97, respectively. Therefore, this is a suitable method for the simultaneous estimation of Montelukast and Doxofylline in drug mixture and dosage forms.

3.2. System Suitability. Five injections of the standard mix were injected for this purpose. The retention time, areas, resolution, theoretical plates values, and peak Asymmetry were calculated for standard. Percentage RSD value was calculated. The Results obtained are given in following Table 1.

3.3. Linearity. The correlation coefficient (r) obtained was calculated and it was found to be greater than 0.99 for Montelukast and Doxofylline, which is well within the acceptance criteria. The results are shown in Table 2. The concentration was found to be proportional to the area and the response of the detector was determined to be linear over the range of 
TABLE 4: Robustness results.

\begin{tabular}{|c|c|c|c|}
\hline & & Retention time \%RSD & Area \%RSD \\
\hline \multicolumn{4}{|c|}{ Different flow rates } \\
\hline \multirow{2}{*}{$0.8 \mathrm{~mL} / \mathrm{min}$} & Montelukast & 0.07 & 0.48 \\
\hline & Doxofylline & 0.15 & 0.16 \\
\hline \multirow{2}{*}{$1.2 \mathrm{~mL} / \mathrm{min}$} & Montelukast & 0.12 & 0.31 \\
\hline & Doxofylline & 0.24 & 0.39 \\
\hline \multicolumn{4}{|c|}{ Different $\mathrm{pH}$ values } \\
\hline \multirow{2}{*}{4.3} & Montelukast & 0.29 & 0.98 \\
\hline & Doxofylline & 0.51 & 0.46 \\
\hline \multirow{2}{*}{4.7} & Montelukast & 0.1 & 0.99 \\
\hline & Doxofylline & 0.24 & 0.24 \\
\hline \multicolumn{4}{|c|}{ Different wave lengths } \\
\hline \multirow{2}{*}{$278 \mathrm{~nm}$} & Montelukast & 0.32 & 0.52 \\
\hline & Doxofylline & 0.12 & 0.1 \\
\hline \multirow{2}{*}{$282 \mathrm{~nm}$} & Montelukast & 0.29 & 0.76 \\
\hline & Doxofylline & 0.38 & 0.49 \\
\hline \multicolumn{4}{|c|}{ Different temperatures } \\
\hline \multirow{2}{*}{$39.5^{\circ} \mathrm{C}$} & Montelukast & 0.09 & 0.34 \\
\hline & Doxofylline & 0.19 & 0.15 \\
\hline \multirow{2}{*}{$40.5^{\circ} \mathrm{C}$} & Montelukast & 0.03 & 0.15 \\
\hline & Doxofylline & 0.06 & 0.21 \\
\hline
\end{tabular}

TABLE 5: \%RSD of the drugs on different days and different analysts.

\begin{tabular}{ccc}
\hline & Retention Time \%RSD & Area \%RSD \\
\hline Day 1 \& analyst 1 & & \\
Montelukast & 0.29 & 0.8 \\
Doxofylline & 0.12 & 0.34 \\
Day 2 \& analyst 2 & & \\
Montelukast & 0.09 & 0.49 \\
Doxofylline & 0.11 & 0.41 \\
\hline
\end{tabular}

TABLE 6: Estimation of the drugs in commercial samples.

\begin{tabular}{lccc}
\hline Telekast-D & Label claim & Acquired data & \%Recovery \\
\hline Montelukast & $10 \mathrm{mg} / \mathrm{tab}$ & $10.03 \mathrm{mg} / \mathrm{tab}$ & $100.30 \%$ \\
Doxofylline & $400 \mathrm{mg} / \mathrm{tab}$ & $399.48 \mathrm{mg} / \mathrm{tab}$ & $99.87 \%$ \\
\hline
\end{tabular}

0.2 to $0.6 \mathrm{mg} / \mathrm{mL}$ for Montelukast and 0.5 to $1.5 \mathrm{mg} / \mathrm{mL}$ for Doxofylline as shown in the Figure 6.

3.4. Accuracy. The results indicate that the recoveries are well within the acceptance range of $80-120 \%$; therefore, method is accurate and it can be used for the simultaneous estimation of Montelukast and Doxofylline. The percentage recovery of the results obtained are listed in Table 3.

3.5. Robustness. Due to deliberate change in the chromatographic conditions of the method, like flow rate, $\mathrm{pH}$, wave lengths, and column temperature, excellent performance of the method was observed. This indicates that the method is Robust. The results obtained are shown in Table 4.
3.6. Ruggedness. Data acquired and \%RSD of area and retention time were calculated for various trials and data tabulated in Table 5. Based on the data, it is evident that the method is rugged.

3.7. Performance of the Method on Commercial Formulations. As per the label claim, the commercial tablet, namely, Telekast-D contains $10 \mathrm{mg}$ of Montelukast and $400 \mathrm{mg}$ of Doxofylline. The quantification of both the drugs was done in the formulation and the results have been found to be in the range of 99.8 to $100.3 \%$. This indicates that the method developed by us can be used for the simultaneous estimation of Montelukast and Doxofylline in any of the pharmaceutical dosage forms. The results obtained are as shown below in the Table 6.

\section{Conclusion}

The novel HPLC method developed is sensitive, unique, precise, user friendly, rapid, and reproducible for simultaneous estimation of Montelukast and Doxofylline in bulk mix and pharmaceutical dosages forms. The method was validated as per the ICH Guidelines. It is concluded that this method can be used by the industries and academic institutions for their combination drug estimation, which is fast as well as novel.

\section{Acknowledgment}

The author (G. Nirupa) would like to acknowledge Startech Labs Private Limited, Hyderabad, India, for providing pure drugs of Montelukast and Doxofylline and providing opportunity to carry out the analytical work. 


\section{References}

[1] G. Nirupa and U. M. Tripathi, "RP-HPLC analytical method developmentand and validation for simultaneous estimation of three drugs: Glimepiride, Pioglitazone, and Metformin and its pharmaceutical dosage forms," Journal of Chemistry, vol. 2013, Article ID 726235, 8 pages, 2013.

[2] G. Nirupa and U. M. Tripathi, "RP-HPLC method development and validation for simultaneous estimation of two drugs Nitazoxanide, Ofloxacinandits Pharmaceutical dosage forms," International Journal of Chem Tech Research, vol. 4, no. .2, pp. 775-783, 2012.

[3] G. Nirupa, Method development and partial analytical method validation of antiasthmatic drug-montelukast [M.Phil thesis], Madurai Kamaraj University, Tamilnadu, India, 2008.

[4] I. A. Alsarra, "Development of a stability-indicating HPLC method for the determination of montelukast in tablets and human plasma and its applications to pharmacokinetic and stability studies," Saudi Pharmaceutical Journal, vol. 12, no. 4, pp. 136-143, 2004.

[5] D. V. Bharathi, K. K. Hotha, B. Jagadeesh, R. Mullangi, and A. Naidu, "Quantification of montelukast, a selective cysteinyl leukotriene receptor (CysLT1) antagonist in human plasma by liquid chromatography-mass spectrometry: validation and its application to a human pharmacokinetic study," Biomedical Chromatography, vol. 23, no. 8, pp. 804-810, 2009.

[6] S. S. Patil, S. Atul, S. Bavaskar, S. N. Mandrupkar, P. N. Dhabale, and B. S. Kuchekar, "Development and statistical validation of spectrophatometry method for estimation of Montelukast in bulk and tablet dosage form," Journal of Pharmacy Research, vol. 2, no. 4, pp. 714-716, 2009.

[7] R. M. Singh, P. K. Saini, S. C. Mathur, G. N. Singh, and B. Lal, "Development and validation of a RP-HPLC method for estimation of montelukast sodium in bulk and in tablet dosage form," Indian Journal of Pharmaceutical Sciences, vol. 72, no. 2, pp. 235-237, 2010.

[8] R. Gannu, S. Bandari, S. G. Sudke, Y. M. Rao, and B. P. Shankar, "Development and validation of a stability-indicating RP-HPLC method for analysis of doxofylline in human serum. Application of the method to a pharmacokinetic study," Acta Chromatographica, no. 19, pp. 149-160, 2007.

[9] A. Mittal and S. Parmar, "Development and validation of rapid HPLC method for determination of doxofylline in bulk drug and pharmaceutical dosage forms," Journal of Analytical Chemistry, vol. 65, no. 3, pp. 293-297, 2010.

[10] A. Gupta, S. Rawat, M. Gandhi, and J. S. Yadav, "Method development and acid degradation study of doxofylline by RP-HPLC and LC-MS/MS," Asian Journal of Pharmaceutical Analysis, vol. 1, no. 1, pp. 10-13, 2011.

[11] A. V. V. N. K. S. Kumar, S. V. Saradhi, C. B. Sekaran, and T. V. Reddy, "Development and validation of novel analytical methods for estimation of doxofylline in bulk and dosage forms," European Journal of Chemistry, vol. 2, no. 3, pp. 372-377, 2011.

[12] S. . Venkatesan, P. Giriraj, S. Myvizhi, P. Kathiravan, and R. singh, "A simple HPLC method for quantitation of doxofylline in tablet dosage form," International Journal of Chemical and Pharmaceutical Sciences, vol. 1, no. 2, pp. 54-57, 2010.

[13] B. R. Challa, B. Z. Awen, B. R. Chandu, M. Khagga, and C. B. Kotthapalli, "Method development and validation of montelukast in human plasma by HPLC coupled with ESI-MS/MS: application to a bioequivalence study," Scientia Pharmaceutica, vol. 78, no. 3, pp. 411-422, 2010.
[14] N. Rashmitha, T. J. S. Raj, C. H. Srinivas et al., "A validated RP-HPLC method for the determination of impurities in montelukast sodium," E-Journal of Chemistry, vol. 7, no. 2, pp. 555-563, 2010.

[15] A. Gupta, V. Yadav, J. S. Yadav, and S. Rawat, "An analytical approach of doxofylline: a review," Asian Journal of Pharmaceutical Analysis, vol. 1, no. 4, pp. 67-70, 2011.

[16] A. S. Rathore, L. Sathiyanarayanan, and K. R. Mahadik, "Development of validated HPLC and HPTLC methods for simultaneous determination of levocetirizine dihydrochloride and montelukast sodium in bulk drug and pharmaceutical dosage form," Pharmaceutica Analytica Acta, vol. 1, p. 106, 2010.

[17] B. V. V. S. Kumar, P. Mathur, N. Rajesh, D. N. Rao, and P. Satyanarayana, "Analytical method development and validation of Levocetirizine hydrochloride and Montelukast sodium in combined tablet dosage form by RP-HPLC," International Journal of Advances in Pharmaceutical Research, vol. 2, no. 7, pp. 380-396, 2011.

[18] S. Ashokkumar, M. S. Raja, and P. Perumal, "RP-HPLC method development and validation for simultaneous estimation of montelukast sodium and levocetirizine dihydrochloride," International Journal of Pharmaceutical Research, vol. 1, no. 4, pp. 8-12, 2009.

[19] A. Basu, K. Basak, M. Chakraborty, and I. S. Rawat, "Simultaneous RP-HPLC estimation of Levocetirizine Hydrochloride and Montelukast Sodium in tablet dosage form," International Journal of PharmTech Research, vol. 3, no. 1, pp. 405-410, 2011.

[20] S. Patil, Y. V. Pore, B. S. Kuchekar, A. Mane, and V. G. Khire, "Determination of montelukast sodium and bambuterol hydrochloride in tablets using RP HPLC," Indian Journal of Pharmaceutical Sciences, vol. 71, no. 1, pp. 58-61, 2009.

[21] S. A. Patel, S. K. Patel, D. J. Patel, and N. J. Patel, "Analytical method development and validation of montelukast sodium and bambuterol hydrochloride in combined dosage form by RPHPLC," International Journal of PharmTech Research, vol. 2, no. 3, pp. 1767-1771, 2010.

[22] D. J. Patel, S. A. Patel, and S. K. Patel, "simultaneous determination of montelukast sodium and bambuterol hydrochloride in tablet dosage form by ultraviolet spectrophotometry (dual wavelength method)," International Journal on Pharmaceuticaland Biomedical Research, vol. 1, no. 3, pp. 71-75, 2010.

[23] R. Revathi, T. Ethiraj, P. Thenmozhi, V. S. Saravanan, and V. Ganesan, "High performance liquid chromatographic method development for simultaneous analysis of Doxofylline and Montelukast sodium in a combined form," Pharmaceutical Methods, vol. 2, no. 4, pp. 223-228, 2011.

[24] S. K. Shetty, K. V. Surendranath, J. Satish, and J. Jogul, "Determination of Zaleplon in the presence of its degradation productsbya stability indicating UPLC method," Analytical Chemistry, vol. 8, no. 3, pp. 360-367, 2009.

[25] S. K. Shetty, K. V. Surendranath, R. K. Kaja, J. Satish, J. Jogul, and M. Upendra, "Development and validation of a stabilityindicating UHPLC method for assay of felbamate and related substances," Acta Chromatographica, vol. 22, no. 2, pp. 161-172, 2010 . 

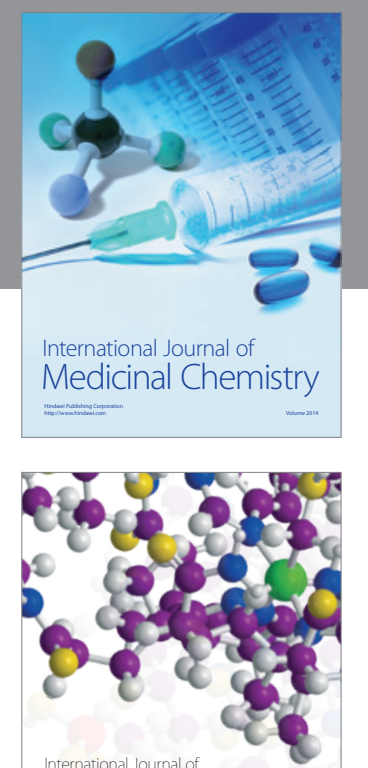

\section{Carbohydrate} Chemistry

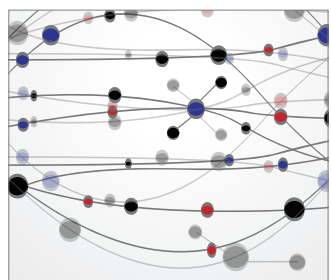

The Scientific World Journal
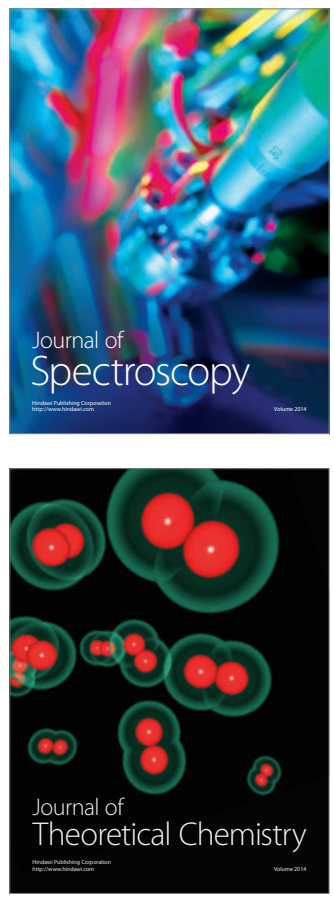
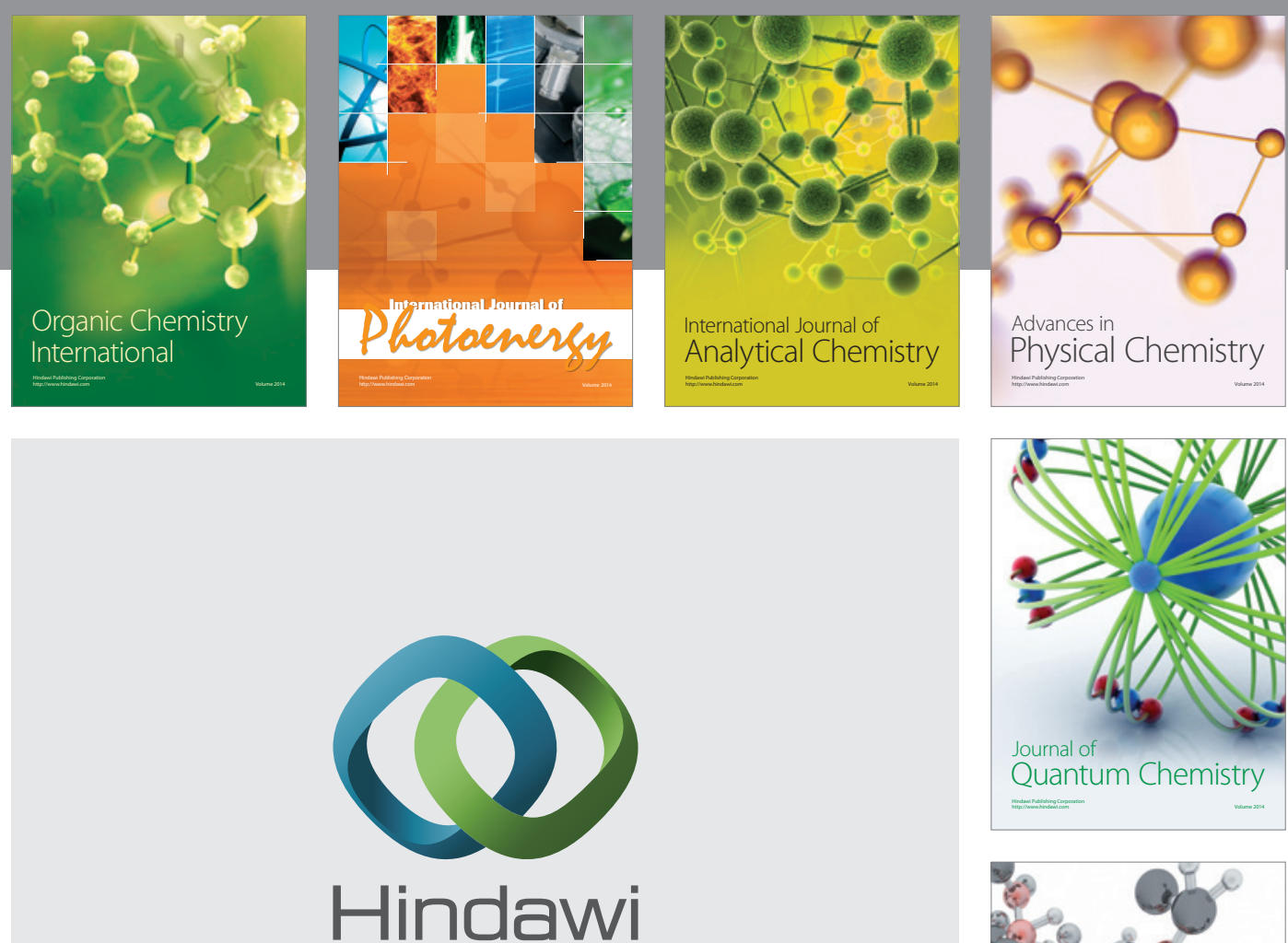

Submit your manuscripts at

http://www.hindawi.com

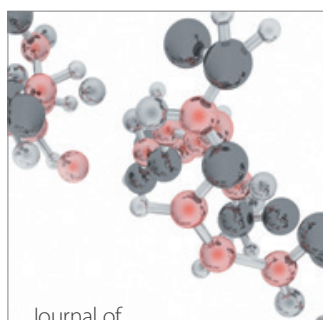

Analytical Methods

in Chemistry

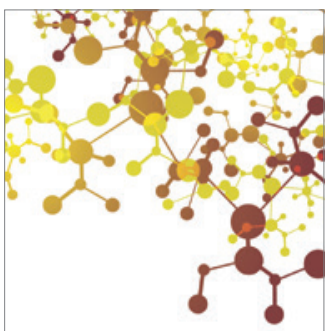

Journal of

Applied Chemistry

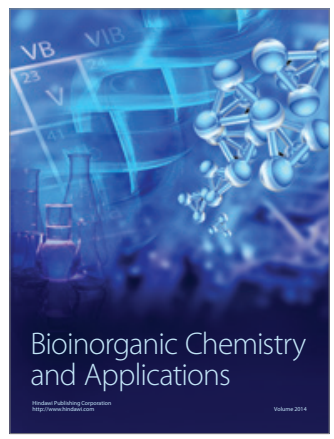

Inorganic Chemistry
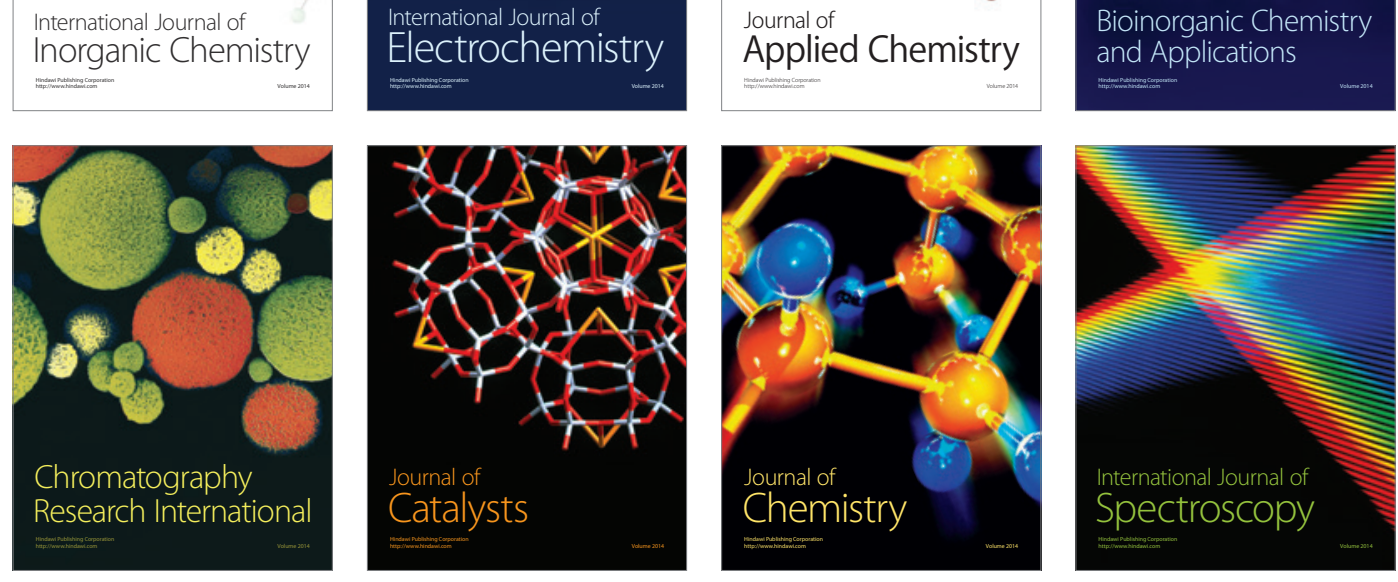